\title{
113. A Method of Raising the Critical Speed for Wing Flutter.
}

\author{
By Katsutada Sezawa, Satosi Kubo \\ and Hirosi MIYAZAKI.
}

Aeronautical Research Institute, Tokyo Imperial University.

(Comm. by. A. Tanakadate, M.I.A., Dec. 12, 1936.)

The prevalent idea for preventing wing flutter is that the mass balance of an aileron or some irreversible control of the same aileron is the most effective. From our studies of this problem, we found that it is possible to raise the critical speed for wing flutter to a far greater extent, even beyond that of the case of a fixed aileron, by merely inserting suitable elastic and damping resistances between the main wing and the aileron. As a matter of fact, it is possible even for an aeroplane with fixed aileron to flutter were the machine to change its attitude from one of horizontal flight to steep dive under certain conditions with respect to its centre of gravity and to those of its linear and angular velocities, when it is, then, even possible for the angle of incidence to assume zero or negative value. The importance of raising the critical speed beyond that in the case of a fixed aileron is so obvious that no comment will be needed.

The case here discussed concerns the torsion-aileron type of wing vibrations, when the wing is most liable to flutter. The wing model used in the experiments was of the U.S.A. 35A type of span, $b=34 \mathrm{~cm}$, and chord, $l=10 \mathrm{~cm}$, and it was made to oscillate only in the torsional sense about an axis, $e=1.5 \mathrm{~cm}$, behind the leading edge. An aileron of chord, $l_{A}=2.5 \mathrm{~cm}$ (its centre of gravity is $s_{W}=6.7 \mathrm{~cm}$ behind the torsional axis), extended throughout the whole span of the main wing. By means of this simple construction we were able to compare the experimental result with the mathematical theory,1) namely the solutions of the equations of motion

$$
\begin{aligned}
& \theta_{T} \frac{d^{2} \beta}{d t^{2}}=-E_{T} \beta-D_{T} \frac{d \beta}{d t}-\frac{\rho}{2} b V^{2} l^{2} \frac{\partial C_{m}}{\partial \beta_{0}} \beta^{\prime}-m_{A} s_{A} \frac{d^{2} \sigma}{d t^{2}} s_{W}, \\
& \theta_{A} \frac{d^{2} \sigma}{d t^{2}}=-E_{A} \sigma-D_{A} \frac{d \sigma}{d t}-\frac{\rho}{2} b V^{2} l_{A}^{2} \frac{\partial C_{A}}{\partial \sigma_{0}} \sigma^{\prime}-m_{A} s_{W} \frac{d^{2} \beta}{d t^{2}} s_{A},
\end{aligned}
$$

in which

$$
\beta^{\prime}=\beta+\tau \sigma^{\prime}+\frac{l / 2-e}{V} \frac{d}{d t}\left(\beta+\tau \sigma^{\prime}\right), \quad \sigma^{\prime}=\sigma+\epsilon \beta^{\prime}+\frac{l_{A}}{2 V} \frac{d}{d t}\left(\sigma+\epsilon \beta^{\prime}\right),
$$

where $\beta$ is the angular displacement of the main wing about its torsional axis and $\sigma$ that of the aileron about its hinge axis relative to the main wing, both taken in the sense as usually done in the angle of attack. The numerical constants obtained by the respective preliminary experi-

1) K. Sezawa and S. Kubo: Kep. Aeron. Res. Inst., No, 136 (1936), 120. 
ments are such that $\beta_{0}$ (angle of incidence) $=-5^{\circ}, \theta_{T}=7 \cdot 35.10^{3}, D_{T}=10^{4}$, $E_{T}=3.83 .10^{7}$ (all in C. G. S. units), $\partial C_{m} / \partial \beta_{0}=1.02, \partial C_{A} / \partial \sigma_{0}=0.916$, $\tau=\left(\partial C_{m} / \partial \sigma_{0}\right) /\left(\partial C_{m} / \partial \beta_{0}\right)=0 \cdot 5, \quad \epsilon=\left(\partial C_{A} / \partial \beta_{0}\right) /\left(\partial C_{A} / \partial \sigma_{0}\right)=0 \cdot 36, \rho$ being air density. Our present object was to ascertain the effect of differences in elastic resistance $E_{A}$, viscous damping $D_{A}$, the moment of inertia of aileron mass $\theta_{A}$ (mass $\left.=m_{A}\right)$, distance of the centre of gravity of that aileron behind its hinge $s_{A}$, etc., the variation in these values in the experiments being shown in Table I. Both elastic and damping resistances increase in the alphabetical order of the cases mentioned in

TABLE I.

\begin{tabular}{c|c|c|c|c|c}
\hline Case & $E_{A}$ & $D_{A}$ & $m_{A}$ & $s_{A}$ & $\Theta_{A}$ \\
\hline$a$ & 0 & 0 & 24.5 & $0 \cdot 70$ & $26 \cdot 0$ \\
$b$ & $0 \cdot 207.10^{6}$ & $4 \cdot 35.10^{2}$ & 26 & $0 \cdot 83$ & $36 \cdot 3$ \\
$c$ & $0 \cdot 361.10^{6}$ & $5 \cdot 17.10^{2}$ & 26 & $0 \cdot 83$ & $36 \cdot 3$ \\
$d$ & $0 \cdot 465.10^{6}$ & $5 \cdot 75.10^{2}$ & 26 & 0.83 & $36 \cdot 3$ \\
$e$ & $0 \cdot 992.10^{6}$ & $7 \cdot 22.10^{2}$ & $26 \cdot 5$ & 0.89 & 40.1 \\
$f$ & $1 \cdot 410.10^{6}$ & $8 \cdot 12.10^{2}$ & 28 & 1.05 & $51 \cdot 4$ \\
$g$ & $4 \cdot 930.10^{6}$ & $20 \cdot 80.10^{2}$ & 30 & 1.27 & 66.5 \\
$h$ & $\infty$ & $\infty$ & - & - & - \\
\hline
\end{tabular}

the table. The criticals of the wind speed (or aeroplane speed) $V(\mathrm{~m} / \mathrm{s})$ obtained experimentally for these cases are shown by small circles in Fig. 1. It will be seen that, while the critical speed increased with increase of $E_{A}, D_{A}$, even beyond the critical value in the case of the fixed aileron, the speed under consideration again decreased for too large values of $E_{A}, D_{A}$, its limiting case coinciding with the case $h$ where the aileron is fixed to the main wing.

With a view to obtaining a mathematical confirmation of the foregoing phenomenon, we solved the problem by our theoretical method, ${ }^{1)}$ using the numerical constants (obtained by preliminary experiments) shown in Table I, etc.; the results are marked by dots in Fig. 1. The results in both cases, namely the mathematical and the experimental, fairly agree, though not perfectly.

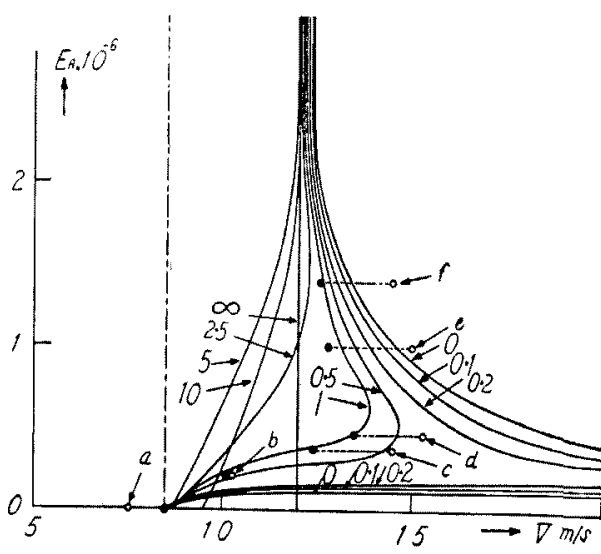

Fig. 1. Critical speed $V$ for different values of $E_{A}$ and $D_{A}$.

1) K. Sezawa and S. Kubo: ibid. 
Although the validity of the problem has been confirmed theoretically, there still remains the question whether it is $E_{A}$ or $D_{A}$ that plays the essential part in the problem, seeing that both $E_{A}$ and $D_{A}$ in Table I vary in the same sense. In order to satisfy ourselves on this point, we calculated mathematically the cases in which $E_{A}$ and $D_{A}$ vary independently; the results are shown by a series of curves also in Fig. 1. The numerals $(0,0 \cdot 1,0 \cdot 2, \ldots, \infty)$ affixed to the respective curves in this figure indicate cases of different $D_{A}$ 's (in C. G. S.) as shown in Table II. Curve $\infty$ corresponds to the case in which the aileron is fixed to the wing, whereas the lowest point of curve 0 (or of the chain line), namely the point at ordinate $E_{A}=0$, corresponds to that in which the aileron is perfectly free. The above result appears to be related in someway to the vibration damper consisting of a coupled oscillator, as recently introduced in mechanical engineering.

Although the fact that the smaller the value of $D_{A}$, for a certain range of $E_{A}$ values, the higher the value of the critical speed invaluably assumed by it, may appear strange, the matter can be explained very simply: in the present model experiment on wing flutter, the aerodynamic damping contributes to a great extent to the damping force, the result being that even in the case $D_{A}=0$, the resultant damping is still too large to raise most effectively the critical speed. In an actual case, on the other hand, as the aerodynamic damping force would be smaller compared with the aileron inertia force. etc., the $D_{A}$ value must have a certain magnitude.

In an actual aeroplane, since the aileron has a very strong elastic resistance owing to that of the control cables, it is practically impossible to use the aileron directly as a vibration damper. On this account it would be advisable for that purpose to fit such an independent elastic oscillator.

TABLE II.

\begin{tabular}{l|ccccccccc}
\hline Curve & 0 & 0.1 & 0.2 & 0.5 & 1 & 2.5 & 5 & 10 & $\infty$ \\
\cline { 1 - 2 }$D_{A} / 5 \cdot 75.10^{2}$ & 0 & 0.1 & 0.2 & 0.5 & 1 & 2.5 & 5 & 10 & $\infty$ \\
\hline
\end{tabular}

After these investigations had been concluded, Mr. Itokawa kindly informed us that aileron shock absorber " repousseau," recently invented by the Repousseau Aviation, and which appears to be fitted to many French aeroplanes, might give rise to a somewhat similar effect in the attempt to prevent wing flutter; but since the absorber in question has to be connected to the control cables in series, the $E_{A}$ value merely corresponds, like the usual shock absorbers, to that at a certain special ordinate in Fig. 1, so that it would hardly be possible to adjust $E_{A}$ to its most suitable condition. According to our present ideas, the repousseau could be made more effective for preventing wing vibrations were it connected to another oscillator, say, one not connected with the aileron. 